\title{
QoS constraint Enhanced AODV in Cognitive Radio Ad Hoc Network
}

\author{
Shobana $\mathrm{M}^{1}$, Vijayanand $\mathrm{M}^{2}$, SanthiyaM ${ }^{3}$, Bharathiraja $\mathrm{N}^{4}$ \\ \{shobanam@srmist.edu.in'1, vijaydeepi1212@gmail.com² ${ }^{2}$, santhiyamunusamy@gmail.com ${ }^{3}$, \\ rajamesoft@gmail.com ${ }^{4}$ \}
}

Assistant Professor, Department of Computer Science and Engineering, SRM Institute of Science and Technology, Kattankulathur ${ }^{1}$, Professor, Department of Computer Science and Engineering, Saveetha Engineering College, Chennai ${ }^{2}$, Assistant Professor, Department of Computer Science and Engineering, Rajalakshmi Engineering College, Chennai ${ }^{3}$, Assistant Professor, Department of Computer Science and Engineering, SRM Institute of Science and Technology, Ramapuram ${ }^{4}$

\begin{abstract}
More range is needed for data communication due to distributed computing, versatility, and the Internet of Things. Regrettably, that range has become a limited resource that is underplayed among licensed clients although these unauthorized selections are overflowing. Intellectual communication creativity is emerged as a potential solution to the range's underutilization. This investigation aims to solve the guiding problem by upgrading the DSR consensus using Quality of Service (QoS) criteria. This investigation determines how such variables affecting QoS could be referred to and in terms with related research. The test employs QoS criteria to improve DSR's display and then evaluate its effectiveness in resolving that steering problem. The proposed plot Q-DSR is compared to the DSR, which is currently in use. NS2 is used to lead reproductions. In terms of speed, temporary suspension, and bundle conveyance ratio, the proposed conspire outperforms the DSR agreement. The strategy worked out how to solve the steering problem yet has gradually reduced the factors that influence QoS. This same disadvantage of said proposed conspiracy is because there is no part to repair disrupted communications after a link break that is being addressed through our future studies.
\end{abstract}

Keywords: Dynamic Source Routing, Cognitive Radio Ad-hoc Network, Quality of Service.

\section{Introduction}

The cognitive radio ad hoc network (CRAHN) is an organization that can adjust and identify naturally the accessible channels. Intellectual radios are fit for changing their arrangements to adjust to the changing condition [1][2]. There are different radio ranges, for example, the worldwide framework for versatile (GSM), TV, remote neighborhood, defense environment, and extended haul advancement as portrayed in figure 1. Some of it is underutilized, while the open classes were overcrowded [3]. The Television and Defense spectrum classes were underutilized to a large extent. 


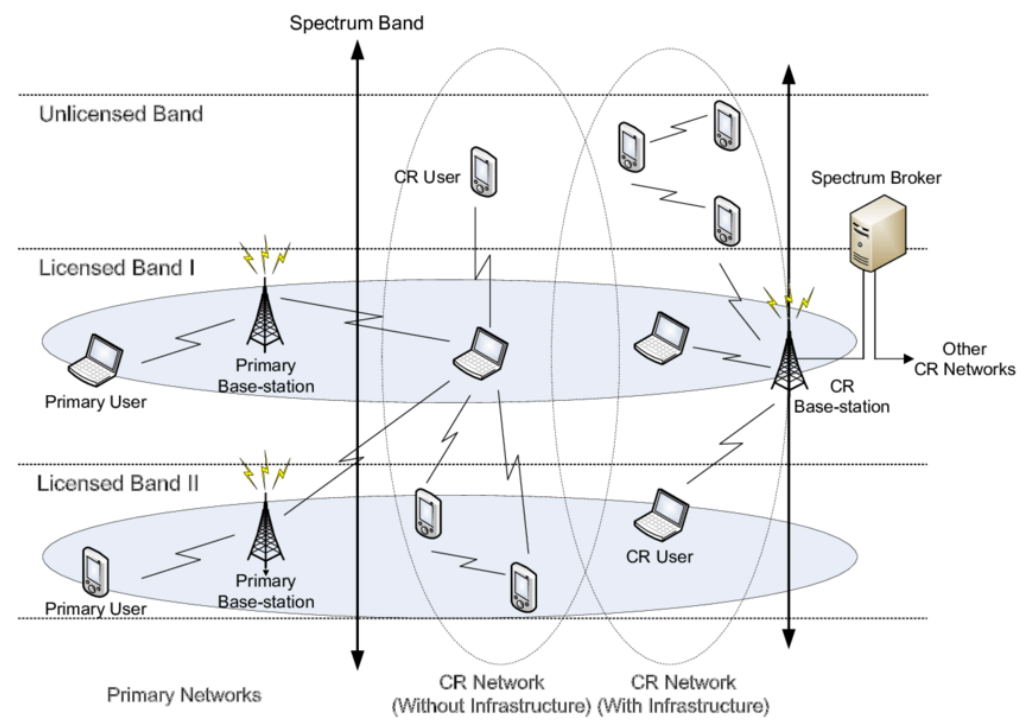

Figure 1: The diagram depicts the distribution of bands among numerous uses.

Cognitive communication may emerge as a potential solution to the problem of underutilized spectrum that can be used by unauthorized clients because when necessary clients remain inactive. Be that as it may, if the PU needs to utilize its range, the optional clients ought to abandon the range without intruding on the PUs exercises. The nature of administration (QoS) in the directing of CRAHNs ought to be thought about given the everexpanding remote gadgets requiring range which brings about range blockage. The breakage of connections and continuous requirement for course disclosure to fix the courses or to find new courses is a test that influences the exhibition of CRN. Nonetheless, in several-bounce organizations, selecting hand-off hubs for high lingering energy enhances this essence between steering. Specially appointed organizations are utilized broadly in a debacles, salvage, vehicular organizations, battle, uneven regions, fire episodes, quakes, and in acoustic submerged organizations as portrayed in figure 2. Booking methods intended to improve the QoS in impromptu organizations are utilized in this investigation to directing proficiency. A decent booking procedure treats the different streams reasonably. This examination considers a few booking methods intended to improve QoS, for example, the FIFO lining, need lining, and weighted reasonable queueing. 


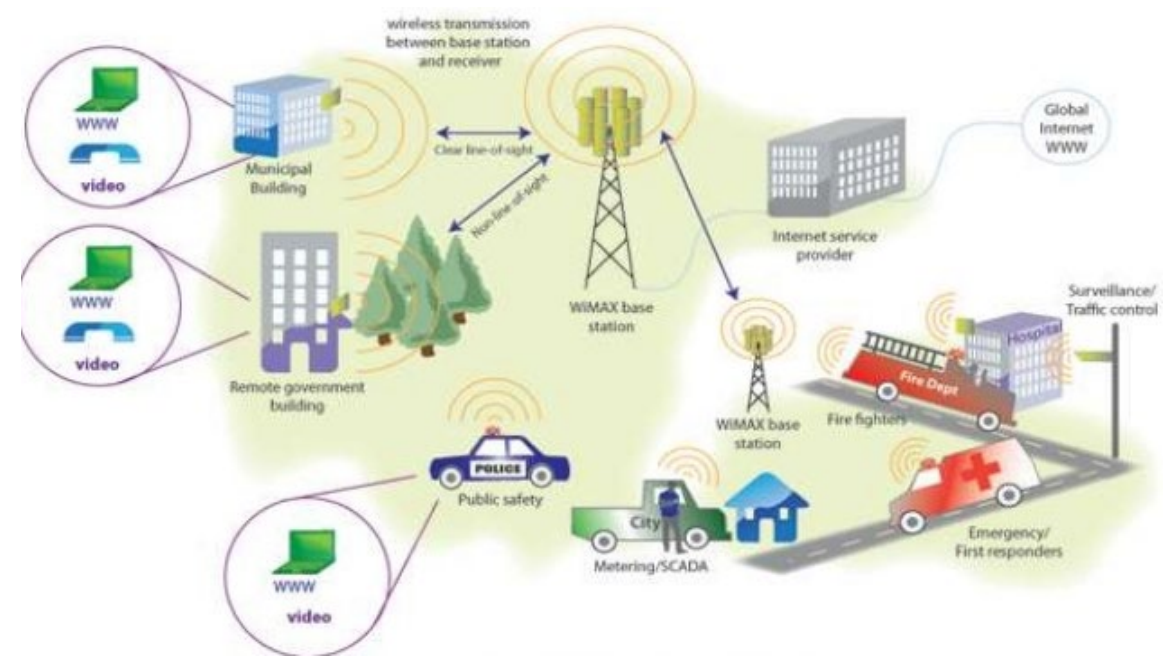

Figure 2: Applications that use cognitive radio networks

\section{Literature Review}

QoS relay nodes look for pathways with enough resources to meet the flow's QoS constraints. Routing protocols that are conscious of QoS should choose routes that are resource-efficient [4]. [5] Looks at certain route selection criteria that are subject to QoS specifications. [6] Proposes a method for auto repairing and enhancing routedesignin ad hoc organization.

In [7], the receptive steering norms are picked because of limited control overheads and expandability yet they experience standard way breakdowns due to expanded relocation of the hubs. For limiting the association breakdowns and to secure a steady way a new responsive directing standard is planned dependent on progressive system based relocations. The expected relocation and scope mindfully appointed on request distance vector steering practice support to resolve the removals including scale highlights over its impromptu organizations. The DDC - AODV coordinates the way distinguishing proof and way affirmation dependent on the uprooting of the contributing hubs and their sizes.

In article[8], authors Shahenda Sarhan and Shadia Sarhan proposed energy effective steering convention dependent on the notable Ad Hoc On-Demand Multipath Distance Vector (AOMDV) directing convention and a bio-motivated calculation called Elephant Herding Optimization (EHO). Inside the suggested EHO-AOMDV, hubs' overall consumed energy is increased by categorizing them within two categories, and routes were found again from group of a best fitted hubs having sufficient power for communication to reduce the probability of way dissatisfaction as well as the growing number of casualties hubs due to increased information loads. After each transmission round, the EHO refreshing administrator refreshes skills based upon on isolating administrator's assessment of hubs based on power consumption.

Authors Srinivas Sethi and Sangita Pal discuss that CRAHN is a consuming innovation in the remote correspondence region and has the high level highlights such as self-recuperating, self-arranging and strength with low arrangement cost. In this climate steering has a 
significant job to build up the way and send the information from source hub to objective hub. As a result, cutting-edge analysis on effective and efficient course creation in organizations is gaining traction.Picking the right peer hub besides sending those bundles will lead to a productive and powerful path.The successful course has been set up in journal [9], using fluffy reasoning based on hub's power and antenna sign power of a hub for greater selection the executives that are major limitations to steering their parcels.

In [10], Thong Nhat Tran etal., proposed a profound support learning-based nature ofadministration steering convention to build up the best course with least start to finish lining postpone subject to the quantity of jumps requirement in psychological versatile impromptu organizations (CRAHNs). In sending RREQ measure, in light of the proposed profound support learning system, the DQR convention route the RREQ bundle to the peered hub with least expense esteem that would save control outgoings, lining lag, and guiding postponement, it stays away from the critical client's influenced location.

Steering in CRAHN is a difficult undertaking because of restricted range accessibility. To defeat this issue a few scientists have proposed different directing plans dependent on QoS and range accessibility. This plans choose a method that provides the highest level of QoS and range, ignoring the required Fault tolerance rate for such a particular function, that might not be on highest quality. In the work, Hardik Dhingra et al suggested an eight-category coordinated support model [11] for CRAHN to imagine confirmation monitoring across Primary User (PU) hubs, thus reducing burden on Secondary Users (SU) for diverse products. To assist Elastic and Real Time Frameworks through (SU) hubs, the PU hub first inspects their range accessibility and then examines that they got package can be enabled client explicit Quality of Service (QoS) or not. The suggested plot correlation with the Cognitive Ad hoc Onrequest Distance Vector or the most restricted range conscious way guiding scheme was completed to demonstrate the effectiveness of the suggested plot correlation. The outcome displays that elite rate for great dependability, low inactivity and high throughput with reasonable burden appropriation among every one of the hubs of the organization.

In [12], creators introduced a postponement and energy-based (DEB) directing convention for the intellectual radio specially appointed organizations. In this work, a bunching approach is considered to partition the organization, where the group arrangement depends on spatial varieties of the range accessibility. When the bunch based organization is framed, the proposed convention empowers any source hub to look and set up a productive course to the objective hub. Characterized as a weighted diagram issue, the proposed DEB steering convention believes postponement and energy to be the directing measurement. In this way, a connection weight is estimated through exchanging and lining deferral of the hubs alongside their remaining energy. It is expected that the proposed convention chooses stable ways while guaranteeing quick information conveyance. The exhibition of the DEB convention has been evaluated through reproduction and contrasted the outcomes and existing conventions. It is seen that DEB displays better execution by outperforming different conventions.

Psychological Radio is indeed a Wi-Fi communicational system which allows clients to communicate with others without being restricted to a specific wireless spectrum. Cognitive Wireless Networks are experiencing difficulties with steering, which is among the important understanding. Specially designated organizations are non-brought together Wi-Fi systems which can be created, and no prior foundation is required for such organizations. Each point will act mostly as switch in this situation. The makers have explained the Radio Resource Technologies which are gaining so much acclaim, with the primary focus on the one-of-a-kind endeavor through streams to remote gadgets. Psychological radio organizations are principally engaged. These days, practically every one of the organizations depends on fixed distributed 
organizations in an affirmed or unapproved recurrence gathering. In [13], Lolita Singh and Nitul Dutta writing assesses related to $\mathrm{CRN}$ and an advancement calculation to upgrade the general presentation of TE under CRN has been talked about. Multitude insight method is utilized in the work. The multitude solution involves unmistakably a combination of decentralized and centralized characteristics in order to obtain magnificent and rational arrangements. Nature provides more motivation than non-standard blueprints on a daily basis.Cat swarm is one of the feasible methods that is often used to secure exorbitant precision and low blunder rates, increasing the organization's life expectancy.The results were analyzed using Cat Swarm Optimization, and boundaries such as power use, blockage, workload usage, and the quantity of steering laws have been used to analyze the overall presentation of a calculation.

Among the most challenging challenges in CRAHNs is increasing throughput in an environment with limited range assets and part of foreign.By using adaptive steering calculation thus achieving Simulated Primary Path, clever range usage will reduce throughput corruption. The Simulated Primary Path[14] targets tracking down the most solid way for multi-bounce correspondence between SU within the sight of PU and other meddling Secondary Users. Virtual Path Routing selects the path that ensures optimal link throughput with the least amount of obstruction by combining mutual steering and diverse range exposure with impedance evasion. Two functionality capacities for guiding intellectual organizations are proposed in this section. The primary power supply combines the probabilistic Transceiver Ratio, the PU effect, and the route choice time delay. The second proposed benefit is based on restricting force use in order to extend life of the battery. In terms of speed, Bit Error Rate, and Packet Arrival Latency, the Simulated Path Routing execution is compared to that of other notable works. Automatically Send Routing, according to the findings, improves bit transfer speed, BER, and PAD by avoiding PU zones and mitigating the impedance effects of neighboring SUs. It can be seen that when a force mitigation strategy, such as water-filling, is used, the Simulated Path Routing increases performance. In comparison to the recently recommended programme, such as Gymkhana with proficient force utilization, the BER for Virtual Path Routing with water-filling is also lower.

In paper [15], Khalid A. Darabkh and Oswa M. Amro considered non-time opened psychological radio specially appointed organizations to directing convention without a typical control channel, in this manner clinging to common sense. The control bundles are sent out in multi - hop and unicast modes to accomplish this.

This convention's exhibition is compared to another significant and ongoing convention, Predictive and Knowable Path Selection (PDPS), which uses an exceptional test method, built on the PDPS test system. Thus using all of the directs in the company, the PDPS convention creates two paths between both the source and the target. Strangely, their outcomes are promising regarding throughput. Then again, our convention works whatever number ways as could be expected under the circumstances between the source and objective. Moreover, in our convention, we utilize every one of the directs in the organization. The presentation metric considered is the throughput while our outcomes are far and away superior to those of PDPS convention.

Psychological Radio innovation has been acquainted with tackle the issues of range underutilization and range shortage brought about by inappropriate range the executives strategies. In CRAHNs, the work not unified framework uphold, information directing experiences different difficulties including successive geography changes, heterogeneous range accessibility, and discontinuous availability brought about withexercises of PU. On this work [16], Zamree Che-aron et al alluded to the Robustness Aware Cognitive Ad-hoc Routing 
Protocol, is introduced by a plan that gives strong way to information conveyance. The Expected Path Delay steering parameter utilized on way choice by presenting that applied in the convention. The measurement assesses the connection delay and the impact of bundle misfortune on remote connections. Besides, the convention tries not to make a communication way which utilizes PU divert in PU districts to balance the effect of PU exercises this could basically aim correspondence interferences. The convention likewise together adventures way with wide variation in steering measure to several hop and various-channel courses with end goal of quick course recuperation.

\section{Issues with Routing}

The range accessibility issue emerges because of the static range assignment strategy. With the headway of innovation and the expanding cell phones, the interest for range is expanding. There is likewise a need to streamline, alter, and to configuration new directing conventions to deliver the steering issue to utilize the range adequately while improving the organization execution. The inaccessibility of organization assets, stable courses, and regular connection breakages debases network execution.

In CRAHNs, a need to resolve solution for guiding problem. Continuous link breaks are caused by hubs and range mobility, which corrupts the presentation of guiding conventions. Whenever the course fails, unenforceable packages accumulate within cushions over extended periods of time, causing them to be lost. We recommend that the CRAHN guiding problem be solved by updating the AODV steering convention and implementing QoS imperatives to ensure that only the courses that fulfill the QoS requirements are prioritized.

\section{Routing Methodology}

QoS steering is an essential feature of CRAHNs. QoS guiding is indeed a form of steering that is based upon that availability of necessary assets such as QoS essentials. The three destinations for QoS guiding are as follows:

- Find an achievable way among source and objective. A way with sufficient assets like battery life, quick organization access, transfer speed, and range to fulfill the QoS prerequisites.

- Optimize the utilization of organization data transmission and assets.

- Adapt to arrange blockage, and choke data transfer capacity for lower-need traffic.

\subsection{Summary of service approaches quality}

Bundles from different streams show up at a switch for the handling which requires reasonableness. This reasonableness can be accomplished through the execution of good planning methods. Moreover, strategies intended to improve the QoS, for example, traffic forming isnecessary. Traffic molding is the instrument which control the sum and pace of route flow shipped off in organization like cracked and token container, affirmation control component utilized by the switch to acknowledge or dismiss a stream dependent on predefined boundaries and asset reservation. Assets like cushion, transmission capacity, computation time, and schedule openings. The QoS has been improvised by these assets which held in advance. QoS directing in the specially appointed organization is a test because of organization geography which changes habitually which makes the steering data be flat. A 
QoS mindful steering convention ought to be improved for the postponement, data transmission, jitter, cost, and misfortune proportion.

\subsection{Phase of Route Discovery}

The control bundles in the recommended QoS-AODV are modified to provide nitty gritty QoS detail.The jump search is used to determine which path is the shortest.The RREQ and RREP packages provide details of available QoS related assets in order to meet the best QoS requirements. The course disclosure as well as area measurement are depicted in Figure 3.

The objective hub piggybacks data around a few bounces, the grouping number, and assets accessible on every way on the RREP bundle. At the point, initial bundle generator hubreceives RREP parcels that verify the data with respect to the most brief way, asset accessibility, and soundness of the connection prior to choosing the best course regarding its capacity to meet the QoS necessities. On the off chance that a course doesn't meet QoS prerequisites, other potential courses are assessed. Just the course meets the QoS necessities is chosen.

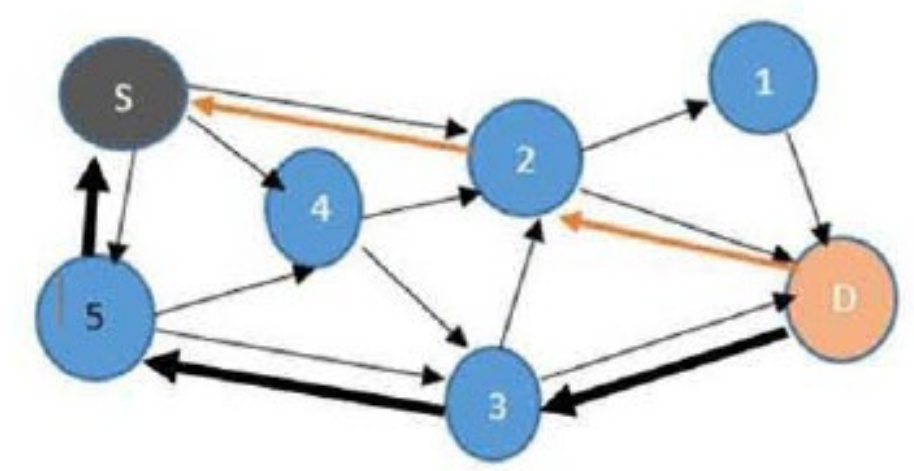

Fig 3: Shows the way revelation measure and the converse way of the proposed plot structure the source hub to the objective hub.

\section{Simulation Metric}

The investigation considered organization geography conveyed in a $1000 \mathrm{x} 1000 \mathrm{~m}$ lattice. The implemented work plot QoS-AODV is assessed and contrasted with the CAODV; this is extended augmentation of AODV directing convention. Table I represents the reenactment boundariesparameter to assess conventions.

The two directing conventions were assessed by difficulties saw in the writing identifying with QoS requirements. The proposed QoS-AODV steering convention QoS mindful. The communication speed was set to run for 100 recreation seconds and the most extreme parcel capacity is set to 100 for support the executives.

Table I displays the parameters that augmentedto compare routing protocols.

\begin{tabular}{|l|l|}
\hline Parameters & Values \\
\hline Simulator & NS 2.35 \\
\hline Routing protocols & QoS-AODV, CAODV \\
\hline Simulation time $(\mathrm{sec})$ & 500 \\
\hline Simulation area & $1000 * 1000$ \\
\hline
\end{tabular}




\begin{tabular}{|l|l|}
\hline Traffic type & CBR/TCP \\
\hline Number of mobile nodes & $10,20,50,80,100$ \\
\hline Transmission Range & $250 \mathrm{~m}$ \\
\hline MAC Type & 802.11 \\
\hline Channel Type & Wireless Channel \\
\hline Antenna Model & Omni \\
\hline Packet Size & 512 bytes \\
\hline Interface Queue Type, length & Drop Tail/PriQueue, 50 \\
\hline Radio Propagation Model & TwoWayGround \\
\hline Data payload & 512 bytes \\
\hline
\end{tabular}

\section{Experimental Solution}

Figure 4 presents the relative normal start to finish postpone results. The outcomes presents the proposed plot has a minimal delay when contrasted with the CAODV convention. The exhibition of QoS-AODV was debased by the expansion in the quantity of hubs. Be that as it may, in comparison to the situation with 100 hubs, its display enhanced for all the number of hubs groups.If a link fails, the CAODV must find alternative methods to avoid prolonged latency issues.

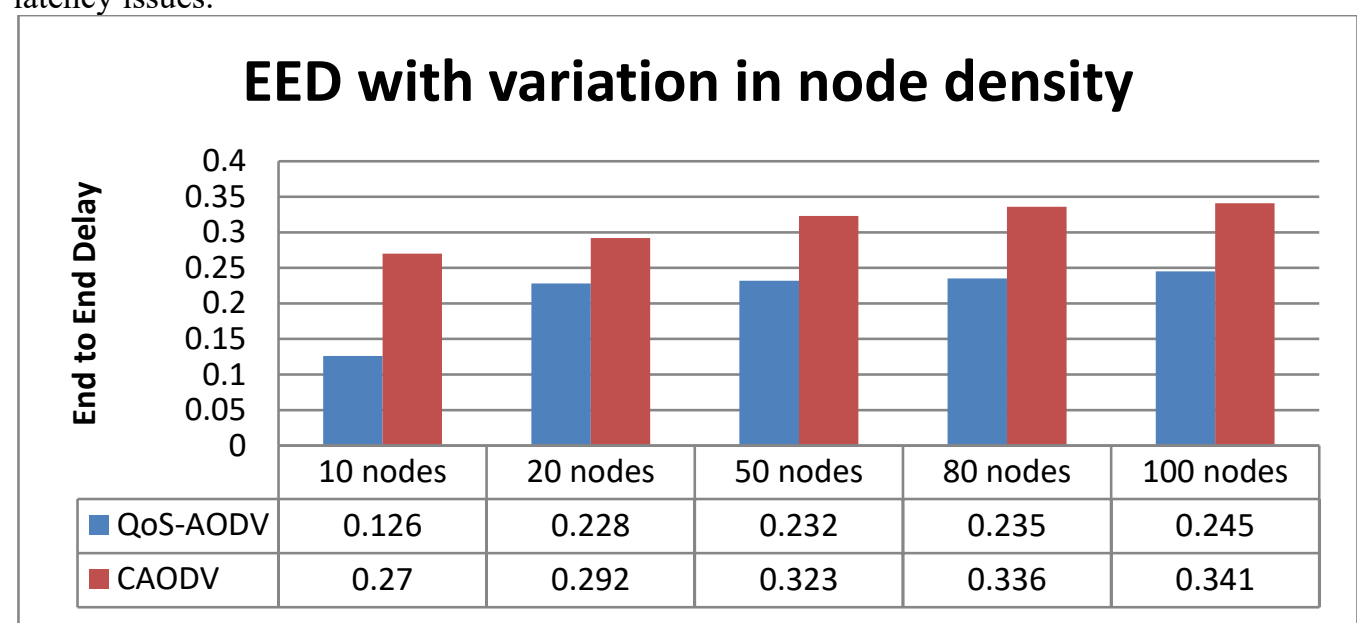

Fig 4: end-to-end delay measures of CAODV compared with QoS-AODV

The presentation of both the guiding conventions for the quantity of fallen parcels is depicted in Figure 5.The results demonstrate that the proposed QoS-AODV plot outperforms the CAODV convention.In any case, the presentation of the two projects in a scenario of 100 hubs is bad, though the QoS-AODV were probably better.The majority of parcels are lost as a result of increased rush hour gridlock with the need to support several bundles over long periods, causing latency issues.Several parcels can be dropped as a result of these deferrals. The QoS prerequisites are in this manner influenced by the quantity of connection breakages that happen as often as possible. 


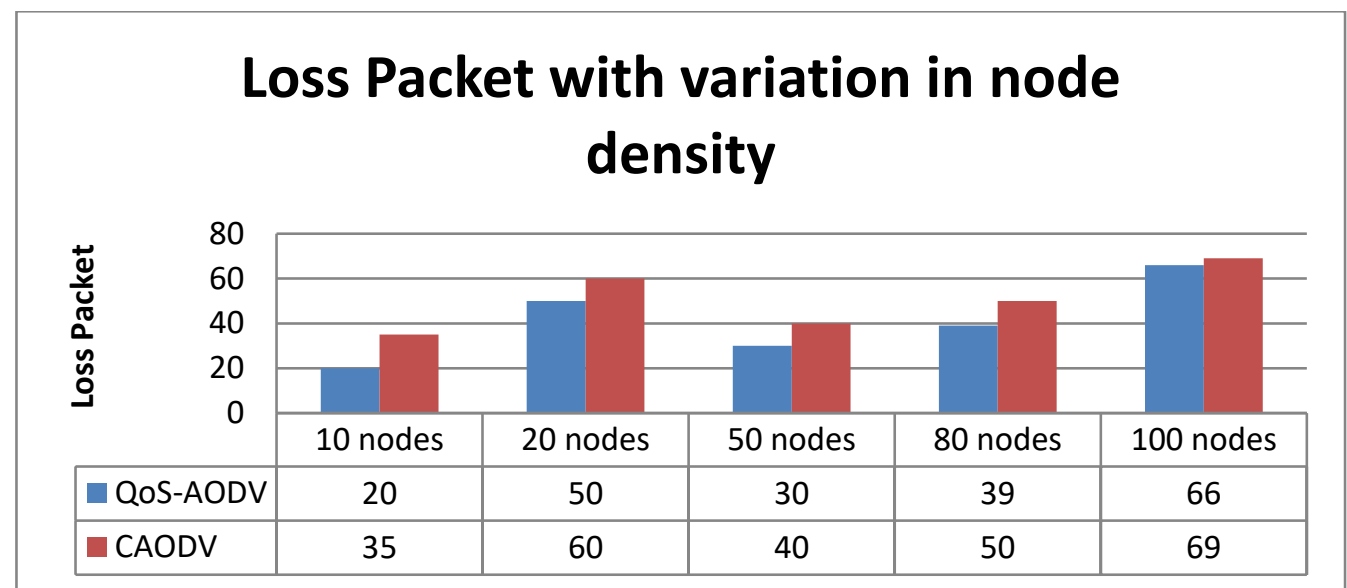

Fig 5: loss Packet delay measures of CAODV compared with QoS-AODV

Figure 6 depicts that conventions' evaluation based on PDR. The QoS-AODV convention outflanked the CAODV convention in terms of parcel conveyance proportion (PDR). The CAODV plot isn't far along in terms of course upkeep.

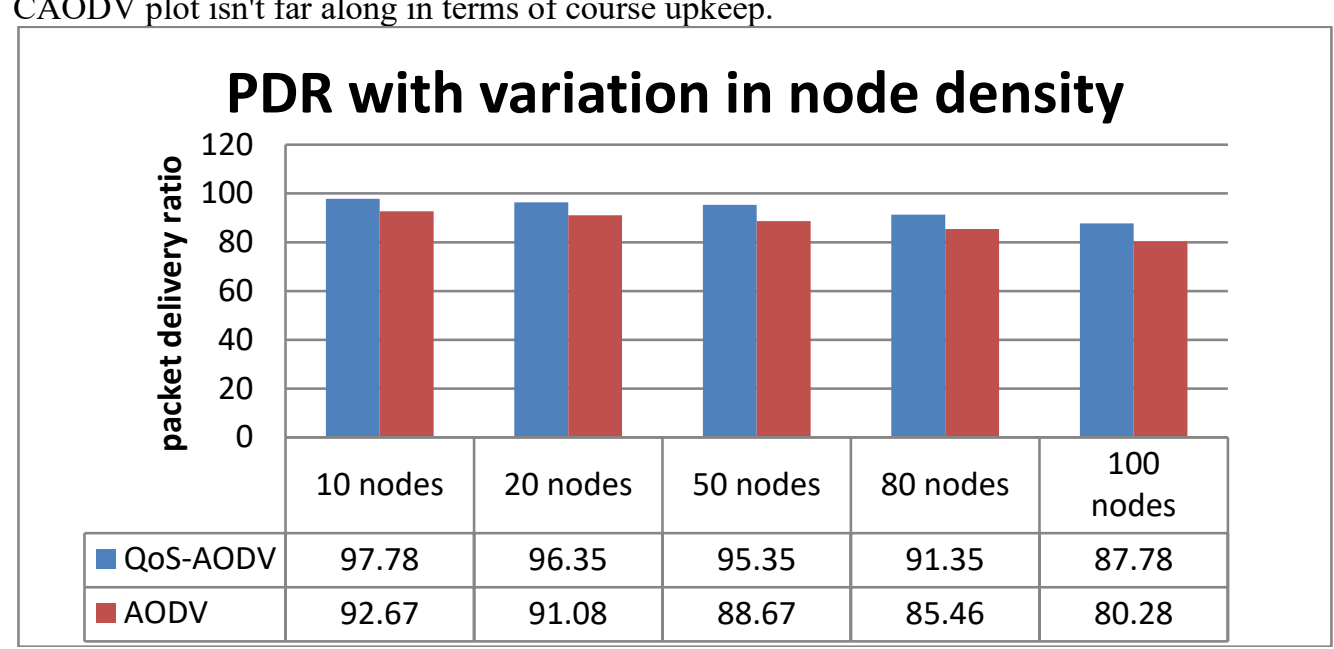

Fig 6: Packet Delivery Ratio measures of CAODV compared with QoS-AODV

Figure 7 examines Throughput and considered as one of the assessment measurements. The similar outcomes show that the feasible throughput of the two conventions expanded with the expansion in the quantity of hubs Notwithstanding. The exhibition of the QAODV convention was prevalent. For a situation with 10 hubs, the QoS-AODV could perform better, and for a situation with 100 hubs, the CAODV organized the results. The QoS-AODV outflanked the CAODV in a variety of cases. The results show that the QoS-AODV convention is successful in determining the best path based on QoS requirements. 


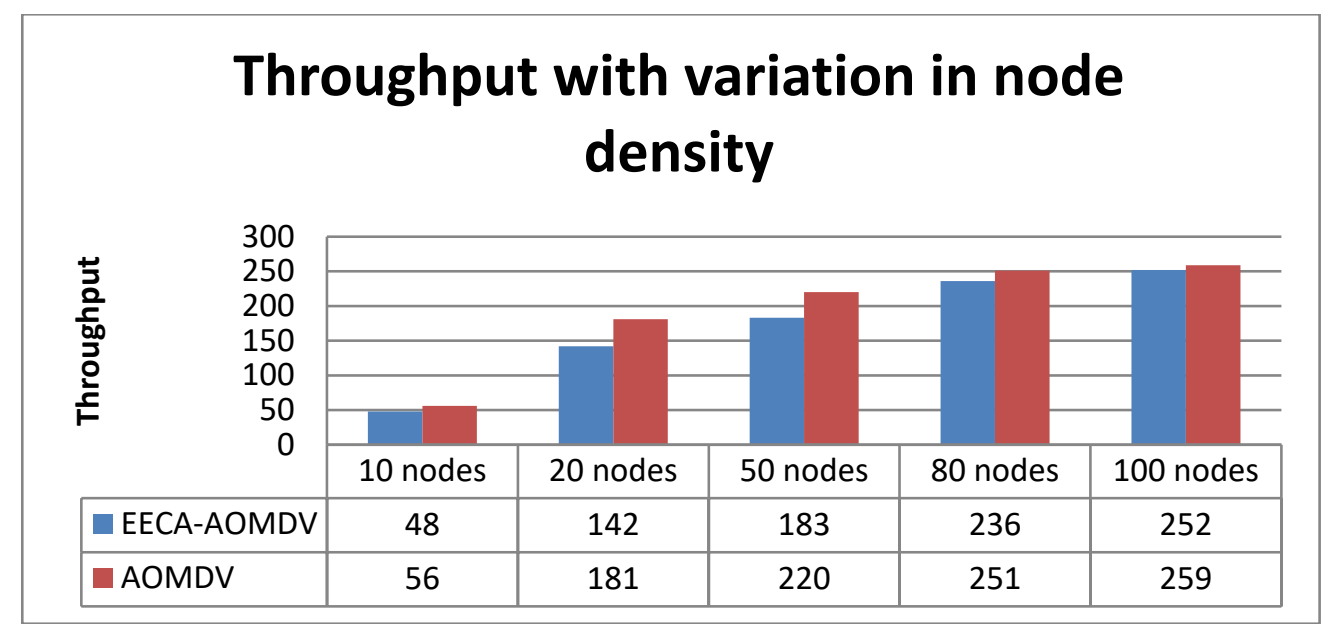

Fig 8: Throughput measures of CAODV compared with QoS-AODV

\section{Conclusion}

The proposed plot has clearly beaten the CAODV steering convention, based on the reenactment results. The plan was improved to reveal secure courses with adequate force that meet the SUs' QoS requirements. This same recreation results showed that the proposal had the potential to overcome the CRAHN steering problem. The NS2 evaluation method was used to evaluate the two conventions' presentations. The steering convention may be changed in the future to aid multiple transmissions. It corrupts in its current structure when exposed to various transmissions. It should be tweaked so that a hub can deal with multiple sources.

\section{References}

[1] S. Kulkarni and S. Markande, "Comparative study of routing protocols in Cognitive Radio Networks," 2015 International Conference on Pervasive Computing (ICPC), Pune, India, 2015, pp. 1-5, doi: 10.1109/PERVASIVE.2015.7087073.

[2] S. Sengupta and K. P. Subbalakshmi, "Open research issues in multi-hop cognitive radio networks," in IEEE Communications Magazine, vol. 51, no. 4, pp. 168-176, April 2013, doi: 10.1109/MCOM.2013.6495776.

[3] "IEEE Standard - Information Technology-Telecommunications and information exchange between systems-Wireless Regional Area Networks-Specific requirements-Part 22: Cognitive Wireless RAN MAC and PHY specifications: Policies and Procedures for Operation in the Bands that Allow Spectrum Sharing where the Communications Devices May Opportunistically Operate in the Spectrum of Primary Service," in IEEE Std 802.22-2019 (Revision of IEEE Std 802.22-2011) , vol., no., pp.1-1465, 5 May 2020, doi: 10.1109/IEEESTD.2020.9086951.

[4] A. A. Junnarkar, Y. P. Singh and V. S. Deshpande, "SQMAA: Security, QoS and Mobility Aware ACO Based Opportunistic Routing Protocol for MANET," 2018 4th International Conference for Convergence in Technology (I2CT), Mangalore, India, 2018, pp. 1-6, doi: 10.1109/I2CT42659.2018.9058022. 
[5] S. Jabbehdari, M. Shamaei and A. Darehshoorzadeh, "IQoS-ODMRP: A novel routing protocol considering QoS parameter in MANET," 2010 IEEE Symposium on Industrial Electronics and Applications (ISIEA), Penang, Malaysia, 2010, pp. 126-130, doi: 10.1109/ISIEA.2010.5679484.

[6] W. Luo, "A quantum genetic algorithm based QoS routing protocol for wireless sensor networks," 2010 IEEE International Conference on Software Engineering and Service Sciences, Beijing, China, 2010, pp. 37-40, doi: 10.1109/ICSESS.2010.5552333.

[7] Saravanan, T., Nithya, N.S. Modeling Displacement and Direction Aware Ad Hoc On-Demand Distance Vector Routing Standard for Mobile Ad Hoc Networks. Mobile Netw Appl24, 1804 1813 (2019). https://doi.org/10.1007/s11036-019-01390-9

[8] S. Sarhan and S. Sarhan, "Elephant Herding Optimization Ad Hoc On-Demand Multipath Distance Vector Routing Protocol for MANET," in IEEE Access, doi: 10.1109/ACCESS.2021.3065288.

[9] Sethi S., Pal S. (2014) Effective Routing Protocol in Cognitive Radio Ad Hoc Network Using Fuzzy-Based Reliable Communication Neighbor Node Selection. In: Satapathy S., Udgata S., Biswal B. (eds) Proceedings of the International Conference on Frontiers of Intelligent Computing: Theory and Applications (FICTA) 2013. Advances in Intelligent Systems and Computing, vol 247. Springer, Cham. https://doi.org/10.1007/978-3-319-02931-3_3

[10] T. N. Tran, T. -V. Nguyen, K. Shim and B. An, "DQR: A Deep Reinforcement Learning-based QoS Routing Protocol in Cognitive Radio Mobile Ad Hoc Networks," 2021 International Conference on Electronics, Information, and Communication (ICEIC), Jeju, Korea (South), 2021, pp. 1-4, doi: 10.1109/ICEIC51217.2021.9369756.

[11] Dhingra, H., Dhand, G.D., Chawla, R. et al. An integrated service model to support user specific QoS routing in cognitive radio ad hoc network. Peer-to-Peer Netw. Appl.14, 18-29 (2021). https://doi.org/10.1007/s12083-020-00965-8

[12] Tasmim S., Kamal A.H., Tusher M.O., Mansoor N. (2020) DEB: A Delay and Energy-Based Routing Protocol for Cognitive Radio Ad Hoc Networks. In: Uddin M.S., Bansal J.C. (eds) Proceedings of International Joint Conference on Computational Intelligence. Algorithms for Intelligent Systems. Springer, Singapore. https://doi.org/10.1007/978-981-15-3607-6_51

[13] Singh L., Dutta N. (2020) A Novel Approach for Better QoS in Cognitive Radio Ad Hoc Networks Using Cat Optimization. In: Sharma N., Chakrabarti A., Balas V. (eds) Data Management, Analytics and Innovation. Advances in Intelligent Systems and Computing, vol 1042. Springer, Singapore. https://doi.org/10.1007/978-981-32-9949-8_42

[14] Mahmud F., Minhas Q., Mahmood H., Muhammad Z., Malik H. (2015) Power Aware Virtual Path Routing Protocol for Cognitive Radio Ad Hoc Networks. In: Yang GC., Ao SI., Gelman L. (eds) Transactions on Engineering Technologies. Springer, Dordrecht. https://doi.org/10.1007/978-94-017-9804-4_37

[15] K. A. Darabkh and O. M. Amro, "New Routing Protocol for Half-Duplex Cognitive Radio AdHoc Networks over IoT Environment," 2020 IEEE International IOT, Electronics and Mechatronics Conference (IEMTRONICS), Vancouver, BC, Canada, 2020, pp. 1-5, doi: 10.1109/IEMTRONICS51293.2020.9216340.

[16] Che-aron Z., Abdalla A.H., Abdullah K., Hassan W.H., Rahman M.A. (2015) A Robust OnDemand Routing Protocol for Cognitive Radio Ad Hoc Networks. In: Kim K., Wattanapongsakorn N. (eds) Mobile and Wireless Technology 2015. Lecture Notes in Electrical Engineering, vol 310. Springer, Berlin, Heidelberg. https://doi.org/10.1007/978-3662-47669-7_4 